which include antibodies against immunoglobulin-E, interleukin-5 and interleukin-4 receptor. In COPD the mainstay of treatment is LABA and LAMA, often as a combination inhaler, whereas ICS are only effective in a proportion of patients that also have eosinophilic inflammation. New safe anti-inflammatory treatments are needed to prevent the progression and exacerbations of COPD.

\section{POETRY, MEDICINE AND THE HIPPOCRATES PRIZE}

${ }^{1}$ Michael Hulse, ${ }^{2}$ Donald Singer. ${ }^{1}$ Department of English and Comparative Literary Studies, University of Warwick, Coventry, UK; ${ }^{2}$ Fellowship of Postgraduate Medicine, London, UK

10.1136/postgradmedj-2018-fpm.6

Why should health professionals be interested in poetry? Patients and their families and carers are accustomed to the use of music and painting in therapy and recent years have seen remarkable growth in the use of poetry for therapeutic purposes. Poetry now features in the curriculum for training health professionals, including doctors, nurses and midwives, in many academic centres in Europe, North America and other continents.

The concept is not new. Poetry was used to treat abnormal mood by the Greek doctor Soranus two thousand years ago. ${ }^{1}$ Renewed interest in poetry as therapy during the 20th-century has inspired a growing body of poetry written about serious medical disorders, including psychiatric problems, HIV/AIDS and cancers. ${ }^{1}$

For almost a decade the Hippocrates Initiative has been exploring the relations between the disciplines of poetry and medicine, both through scholarly inquiry at its annual international symposium and creatively through the Hippocrates Prize for Poetry and Medicine, which over the years has attracted entries from some seventy countries worldwide. During this session on poetry and medicine, we shall hear readings from the Hippcrates Prize by previous winners Jane Draycott and Wendy French.

We take great pleasure in acknowledging the generous support of the UK's Fellowship of Postgraduate Medicine (for the health professional and open categories of the Hippocrates Prize) and of UK healthy heart charity the Cardiovascular Research Trust (for the young poets' award). Judges have included eminent poets such as Jorie Graham, Gwyneth Lewis, Philip Gross, Jo Shapcott and the late Dannie Abse, leading figures from the medical profession such as Professor Sir Bruce Keogh, Professor Steve Field, Dr. Owen Lewis and Professor Femi Oyebode, and prominent people from public life, among them James Naughtie, Robert Francis QC, Martha Kearney, Neal Baer and Mark Lawson.

There were entries from thirty-seven countries on five continents for this year's prizes in the open category, the health professional category, and the young poets category. These awards were announced on 11 May at the Poetry Foundation in Chicago following the Hippocrates international symposium on poetry and medicine, hosted this year by the Center for Bioethics and Medical Humanities at Northwestern University's Feinberg School of Medicine.

The 10th annual Hippocrates prizes will be awarded on 17th May 2019 at the Centre for Life in Newcastle, with American-Mexican poet and PEN international President Jennifer Clement judging the international Open and Health Professional awards (closing date 14th February 2019) and New
Zealand poet Elizabeth Smither judging the young poet awards (closing date 1 st March 2019). To find out more about the Hippocrates Prize, Hippocrates Press publications, and the Hippocrates Society, please visit www.hippocrates-poetry.org.

\section{REFERENCE}

1. Singer DRJ, Hulse MW. Poetry, medicine, and the 2018 international Hippocrates Prize. Lancet. 2010 March 20;375(9719):976-7.

\section{NEW APPROACHES FOR OLD DISEASES: WHAT TO DO ABOUT MALARIA?}

Sanjeev Krishna. St George's University of London, UK

10.1136/postgradmedj-2018-fpm.7

Disease and deaths caused by malaria have fallen significantly in the past few years. These successes rely on many interventions such as better diagnosis, the use of insecticide impregnated bed nets and artemisinin combination therapies. There is also hope that vaccines can be improved to assist in elimination programmes that have been so successful in many countries.

Gains against malaria remain fragile because effective interventions can fail quickly. Mosquitoes can be selected for resistance to insecticides, and parasites escape from artemisinin combination treatments. Vulnerable populations such as pregnant women still only have a limited repertoire of preventative strategies available. Whilst malaria caused by conventional species is as old as the hills, zoonotic parasites that infect humans in particular geographic areas are emergent infections.

Some of these risks of increasing malaria burdens can be mitigated by recent advances. The need for more sensitive diagnostic tests in the era of elimination is being addressed, together with newer methods to monitor molecular markers for drug resistance. New drug classes are being discovered, and there are many vaccine candidates in different stages of clinical development. Together with these innovations and increasing resources to manage malaria, there is also greater scope for applying existing antimalarials more effectively.

\section{AGEING AND MULTI-MORBIDITY}

Thomas BL Kirkwood. Newcastle University Institute for Ageing, UK

\subsection{6/postgradmedj-2018-fpm.8}

A growing fraction of the population is living to advanced old age, bringing increased prevalence of a wide range of agerelated chronic diseases. Age is much the largest risk factor for a whole spectrum of different diseases, dwarfing the contributions from genetic, lifestyle and environmental risk factors. Furthermore, the fact that so many conditions share ageing as their dominant risk factor means that very old people commonly exhibit extensive multi-morbidity. In terms of underlying mechanisms, we now know that ageing itself is not programmed. On the contrary, our bodies are programmed for survival, but evolutionary considerations mean that there was never reason to invest in the potential for indefinite survival. Consequently, we now understand that ageing is driven by the lifelong accumulation of damage in our cells and organs, and the same is true of age-related, chronic diseases. Thus, there is a huge overlap. Recognition of this common 